Results Eleven patients were diagnosed of having AVN: Eight male and three female. The mean age was 27.6 years (23-32). The risk factors for HIV infection was as follows: 8 IDU,3 HMX. Nine patients had had at least one opportunistic infection with a mean time of 30.5 months before the diagnosis of the AVN (12-108). The mean $\mathrm{CD}^{+}$and $\mathrm{pVL}$ at AVN were 328.9 $\mathrm{cel} / \mathrm{mm} 3$ (73-900) and 14.964 copies/ml (6 of them had undetectable levels: $<200$ copies $/ \mathrm{ml}$ ) respectively. Mean $\mathrm{CD}^{+}$nadir was $53 \mathrm{cel} / \mathrm{mm} 3$. The mean time on PI-CR before the AVN was 19.4 months ( 2 patients were naïve for PIs before AVN). MRI showed a bilateral femoral head involvement in 9 patients, 2 of them with additional humeral and knee involvement. Other risk factor related to the development of AVN were evaluated: 6 patients had received corticosteroids prior to the AVN, hyperlipidaemia secondary to PI-CR was reported only in 2 patients. Six months before the AVN, salmonella typhymurium bacteriemia was documented in 2 patients.

Conclusion Despite the higher incidence of AVN reported in HAART era, epidemiological studies should be conducted in order to establish the role of PIs in its aetiology. Other risk factor such as corticoesteroids use related to AVN, should be assessed in HIV+ patients with AVN.

\section{THU0222 TRANSIENT BONE MARROW OEDEMA AND/OR TRANSIENT OSTEOPOROSIS OF THE HIP ARE ASSOCIATED WITH GENERALISED DIMINUTION IN BONE MINERAL DENSITY: ROLE OF PRE-EXISTING BONE FRAGILITY ?}

${ }^{1} \mathrm{~T}$ Pham, ${ }^{1} \mathrm{~V}$ Legré, ${ }^{2} \mathrm{P}$ Champsaur, ${ }^{2} \mathrm{C}$ Chagnaud, ${ }^{1} \mathrm{P}$ Lafforgue. ${ }^{1}$ Department of Rheumatology; ${ }^{2}$ Department of Radiology, CHU La Timone, Marseille, France

\subsection{6/annrheumdis-2001.754}

Background Transient osteoporosis of the hip (TOH) and transient bone marrow oedema of the hip (TBMEH) are uncommon, closely associated and/or similar conditions, whose pathophysiology remains obscure. Several facts suggest that occult microfractures of the femoral head could be the cause of such entities.

Objectives We conducted a prospective assessment of bone status in skeletal sites other than the affected hips to test the hypothesis of pre-existing underlying generalised bone fragility.

Methods 13 consecutive patients with typical signs of TBMEH at MRI were enrolled, with careful exclusion of cases presenting with any sign consistent with either osteonecrosis or stress fractures of the femoral head. Five patients also had radiological signs of TOH. All had prospective bone status assessment including search for risk factors of bone fragility or history of fracture, laboratory tests, spine radiographs and lumbar and femoral neck (of the unaffected hip) by DEXA.

Results There were 8 women (6 pregnant) and 5 men, aged 40 \pm 11 years. Four had possible risk factors for osteoporosis, and only 1 had previous insufficiency fracture (rib). Lumbar BMD measurements by DEXA found 1 patient with normal BMD (Tscore between 0 and 1SD), 9 patients had a T-score between 1 and 2.5 SD and 2 had T-score less than 2.5 SD. The BMD values were about $15 \%$ less than the age/sex matched reference population (mean Z-score $1.4 \pm 0.64 \mathrm{SD}$ ). BMD values obtained in the unaffected hip in 6 cases confirmed these results. Separate analysis of males/females and pregnant/other patients yielded the same results.

Conclusion Most patients with TBMEH and/or $\mathrm{TOH}$ have a generalised decrease in BMD. These results put together with data from the literature suggest that a pre-existing bone fragility is a predisposing factor to TBMEH and/or TOH.

\section{THU0223 TREATMENT OF A PATIENT WITH NONCLASSIFIED HLA B27 ASSOCIATED DESTRUCTIVE OLIGOARTHRITIS WITH ANTI TNF: CLINICAL OUTCOME AND EFFECTS ON NF- KAPPA B ACTIVITY IN PBMC}

T Geiler, R Voll, HM Lorenz, C Dechant, B Manger, JR Kalden. Department of Medicine III, University of Erlangen-Nuremberg, Erlangen, Germany

\subsection{6/annrheumdis-2001.755}

Background We report the case of a 38 years old HLA B27 positive male with a 11 year history of a destructive nonclassified oligoarthritis. While the first 6 years of the disease have been dominated by a relapsing nondestructive synovitis of the right knee joint and a asymptomatic sacroileitis treated with NSAID`s alone the disease began to spread in a destructive manner to the right wrist and foot (MTP I-V, PIP I-V, DIP I-V). Additionally, a painful tenosynovitis of the flexor and extensor tendons of both feet occured. Despite intensifying the pharmacological treatment by high doses of corticosteroides (up to $100 \mathrm{mg} / \mathrm{d}$ ) and methotrexate $(25 \mathrm{mg} /$ week) for 9 month the disease showed significant clinical and radiological progress. No reduction of the constantly elevated ESR and CRP could be achieved. By initiating a therapy with anti TNF (Remicade ${ }^{\mathrm{TM}}$ ) at a dose of $5 \mathrm{mg} / \mathrm{kg}$ body weight all clinical symptoms disappeared completely within $8 \mathrm{~h}$ after the first infusion of the antibody. ESR and CRP normalised within 6 days. The patient stayed completely free of symptoms since, side effects have not been detected by now. Repeated infusions after 2, 4, 6 and 14 weeks did not further improve the therapeutic benefit but were given to sustain the therapeutic effect.

Objectives Since TNF is known as a strong activator of NF- $\kappa B$ which is strongly involved in the production of proinflammatory cytokines and matrix metalloproteinases we looked for the activity of this transcription factor in PBMC`s of this patient by electro mobility shift assays (EMSA).

Methods Cells were obtained immediately before the infusions were started and $1 \mathrm{~h}$ and $24 \mathrm{~h}$ after end of infusions. Cell preparations and EMSA were done according standard protocols.

Results There was substantial NF- $\kappa \mathrm{B}$ activity in PBMC`s before first antibody treatment. Anti TNF treatment decreased NF- $\kappa B$ activity detectable already $1 \mathrm{~h}$ after first infusion. The NF- $\mathrm{B}$ activity was further inhibited $24 \mathrm{~h}$ after first infusion and remained low during the whole treatment period in accordance to the persisting clinical improvement.

Conclusion In the treatment of patients with progressive nonclassified HLA B27 oligoarthritis resistent to classical treatment with NSAID`s, corticosteroids and DMARD`s the use of TNF blocking agents should be considered. As in other destructive inflammatory joint diseases NF- $\mathrm{\kappa B}$ might play a crucial role in the pathogenesis of joint destruction in HLA B27 associated arthritides. The clinical improvement appears to be associated with a persistent and marked inhibition of NF-kappa B. 